\title{
Sampling frequency and the study of eye-hand coordination in aiming
}

\author{
WERNER F. HELSEN \\ Katholieke Universiteit Leuven, Leuven, Belgium \\ and \\ JANET L. STARKES, DIGBY ELLIOTT, and KATHRYN L. RICKER \\ McMaster University, Hamilton, Ontario, Canada
}

\begin{abstract}
This study synchronized sampling of point of gaze (PG) and hand movements in a fast aiming task, using a 60 - and a $120-\mathrm{Hz}$ sampling frequency. The subjects moved eyes, head, hand, and trunk freely. For limb kinematics, a significant difference between sampling conditions was only found for the number of accelerations in the profile following peak velocity of the hand. For PG movements, no differences were found for initiation time, saccade angle, fixation duration, and overall number of saccades. However, significant differences were observed for saccade duration. Previously, an invariant feature was found for the ratio of PG and hand response times (50\%). For both sampling frequencies, a significant correlation and, thus, temporal coupling was found between PG response time and time to peak acceleration for the hand. Depending on the measures required, a 60- $\mathrm{Hz}$ sampling of $\mathrm{PG}$ and hand movements may provide as meaningful results as a $120-\mathrm{Hz}$ sampling.
\end{abstract}

In order to model the organization of eye, hand, and head movements in manual aiming and grasping, it is important to be able to measure the movement of each element independently, in three-dimensional (3-D) space, at an adequate sampling frequency. The advent of Watsmart and Optotrak systems (Northern Digital, Waterloo, Ontario), both of which are capable of high-frequency sampling (e.g., 3,500 markers per second for the Optotrak 3000 series) has made this possible in terms of tracking the limb. Combining these measurements with recordings of eye movements has been extremely difficult. Until recently, corneal reflection systems (e.g., NAC eye-mark recorder-600, Tokyo, Japan), as well as systems using both pupil center and corneal reflection (e.g., ASL series 4000 eye trackers, Applied Science Laboratories, Bedford, MA), only permitted sampling frequency of up to $60 \mathrm{~Hz}(50 \mathrm{~Hz}$ in Europe). Although other measurement techniques (i.e., EOG) permit high-frequency sampling of the eye, they do not permit simultaneous natural movements of the head and the trunk. Some techniques that do allow these movements (e.g., the more invasive magnetic field-search coil technique used by Robinson [1963] and modified, more recently, by Radau, Tweed, and Vilis [1994] in order to measure 3-D eye, head, and chest orientations after large

W.H. acknowledges the National Fund for Scientific Research of Belgium for the support of this research project. The participation of J.S. and D.E. was supported by grants from the Natural Science and Engineering Research Council of Canada. The authors thank Pol Meugens and John Moroz for providing invaluable guidance in designing the research equipment and the electronics, and Jeremy Rodney, Travis Ratnam, and Kevin Smith for assistance with the data reduction. Requests for reprints should be sent to W. Helsen, Katholieke Universiteit Leuven, Motor Learning Laboratory, Tervuursevest 101, 3001 Leuven, Belgium (e-mail: werner.helsen@flok.kuleuven.ac.be). gaze shifts) are less acceptable in terms of spatial resolution. This has meant that, if one wants to track both the eye and the limb synchronously, one has to sample data for both at $60 \mathrm{~Hz}$. Sampling at this frequency means that samples are recorded every $16.6 \mathrm{msec}$, providing only gross temporal resolution. Yet, whether this sampling frequency is too gross could never be determined, because an alternative higher sampling frequency was not available for comparison. One alternative has been to sample the eye at $60 \mathrm{~Hz}$ and the limb at a higher frequency, but this meant that sampling could not be synchronized.

Recently, ASL has marketed a high-frequency eye movement analysis system (4100) that still uses pupil center/corneal reflection but samples at $120 \mathrm{~Hz}$. For the first time, this permits synchronized sampling of the eye and limb at acceptable frequencies. It also permits a comparison of the data obtained when the eye and the limb are synchronously sampled at both 60 and $120 \mathrm{~Hz}$. Presumably, if there are big differences in the data acquired at the two sampling frequencies, researchers may be forced to purchase the higher speed system. Alternatively, if no differences are apparent from the data sampled at different speeds, researchers may have some justification for and confidence in the data they currently obtain from $60-\mathrm{Hz}$ systems.

This paper assesses how synchronization of point of gaze (PG) and hand movements is affected by sampling frequency $(60 \mathrm{~Hz}$ vs. $120 \mathrm{~Hz})$ when a subject is required to make multiple-degree-of-freedom aiming movements between two targets.

\section{METHOD}

\author{
Subjects \\ Ten male graduate students, ranging in age from 21 to 28 years \\ (mean age 23.4 years), voluntarily participated in the experiment.
}


All were right-handed and had normal or corrected-to-normal vision, on the basis of self-report.

\section{Task}

The subject was seated at a table (Figure 1), with his right index finger on a microswitch at eye level in the frontoparallel plane. A red stimulus light was first highlighted behind the home button, and, when this light went off, the subject had to move to and press the target button located $40 \mathrm{~cm}$ to the right. The movement was to be completed as fast as possible. Target buttons were $1 \times 2 \mathrm{~cm}$. The index of difficulty [ID $=\log _{2}(2 \times$ amplitude/width $\left.)\right]$ for this task was 6.32 bits (Fitts, 1954). The red stimulus light and other environmental cues were easily seen, since ambient luminance during the test was $15 \mathrm{~cd} / \mathrm{m}^{2}$.

Throughout the experiment, the subject wore a single infrared emitting diode (ired) attached to the right index finger on an adjustable metal band. He also wore a head-mounted eye tracker $(750 \mathrm{~g})$ fastened on a hockey helmet. Counterweights distributed the weight of the helmet and eye tracker evenly over the head.

\section{Apparatus}

Finger kinematics: Optotrak-3020 analysis. A wall-mounted Optotrak 3-D movement analysis system provided information about the position of the single ired attached to the forefinger. The Optotrak covers a test area $2 \mathrm{~m}$ high $\times 3.4 \mathrm{~m}$ wide at $6 \mathrm{~m}$ with a spatial accuracy of $0.3 \mathrm{~mm}$. A standard IBM/AT- 486 compatible computer supported the system.

Point of gaze tracking: ASL $\mathbf{4 1 0 0}$ analysis. Eye movement recordings were made with an ASL 4100 head-mounted eye tracker. This system uses the pupil center/cornea reflection method (Young \& Sheena, 1975) and allows normal head movements. The eye tracker was combined with an independent measure of head position and orientation (from a magnetic head-tracking system that included a reference source [MHT transmitter] located behind the subject and a small sensor [receiver] fastened to the helmet). This permitted the use of digital tracking of PG in the environment. The MHT covered a test area $1 \mathrm{~m}^{3}$, with a spatial accuracy of $0.6 \mathrm{~mm}$. A Pentium 133-MHz computer served as the processor for computing eye/head integration. It also served as the user interface for eye tracker operation, data recording, and analysis. The subject could freely move his head throughout the experiment; thus, when the subject looked straight ahead, the visual angle between the home position and the target button varied according to the subject's eye-to-scene distance (e.g., $40^{\circ}$ of visual angle for $55 \mathrm{~cm}$, measured from an imaginary point halfway between the eyes).

\section{Procedure}

Both the aiming stimulus and the subject's elbows were positioned on a table so that the head was kept in horizontal alignment with the movement task. Each subject received standardized instructions.

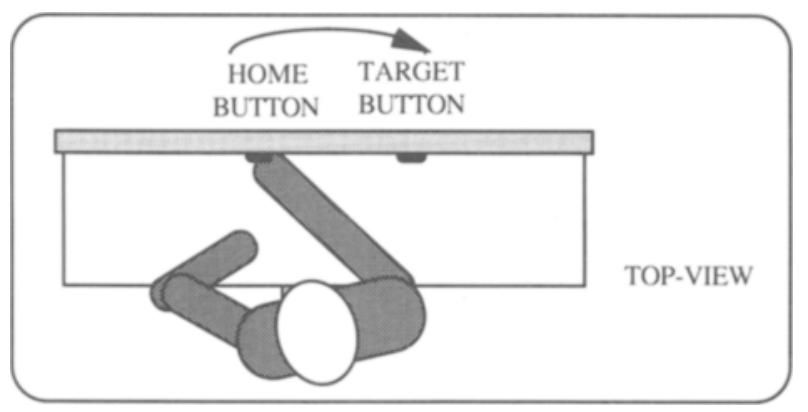

Figure 1. The experimental test set-up.
First, the Optotrak system was referenced using three ireds placed at the same height as the actual home and target buttons. Then the eye movement recording apparatus was fitted onto the subject's head and stabilized. It was calibrated for both position and linearity to ensure that the fixation mark corresponded precisely to the subject's visual orientation to nine different sectors of a calibration screen. Throughout the test, the subject was instructed to return visual focus to the home position after each response.

Testing at $60 \mathrm{~Hz}$ and at $120 \mathrm{~Hz}$ was conducted on 2 separate days within a week. The procedures each day were identical, and frequency order was counterbalanced across subjects (i.e., 5 subjects in each order).

The subject was told that his task was to move the right index finger as fast as possible from the home button to press the target button, $40 \mathrm{~cm}$ to the right, as soon as the red light behind the home position went off. Presentation time of the stimulus was randomly varied from 2 to $4 \mathrm{sec}$ to avoid anticipation by the subject. In each condition, the subject was allowed to move the eyes, head, and trunk freely when the stimulus light went off. At the beginning of each trial, however, the eyes, head, and hand were all aligned on the home position to ensure spatial compatibility. Target acquisition was assessed on five blocks of five trials. After the first and the third blocks of trials, the subject was provided with a 1 -min rest.

In this study, two experimenters were necessary; while one made sure the ired mounted on the index finger was visible to the Optotrak cameras throughout the movement, the other first ensured that the finger and $P G$ were aligned on the home position before each trial and, second, monitored PG data across trials.

\section{Data Reduction and Analysis \\ Finger kinematics: Optotrak analysis. Using the same proce- dure as that of Chua and Elliott (1993),}

the raw displacement data for each trial were filtered using a secondorder dual-pass Butterworth filter (low-pass cutoff frequency of $6.0 \mathrm{~Hz}$ ). Instantaneous velocity was calculated by differentiating displacement data using a two point central finite difference algorithm. Instantaneous acceleration was calculated by differentiating velocity data using the same algorithm. Since the movement was constrained (primarily) to the horizontal axis, kinematic data were analyzed with respect to this axis only. (pp. 371-372)

Chua and Elliott's (1993) interactive program was used to identify certain critical points in the displacement, velocity, and acceleration profiles for each trial. To define the beginning of a movement, the first occurrence of instantaneous velocity greater than or equal to $50 \mathrm{~mm} / \mathrm{sec}$ was noted. Movement end was defined as the time at which the target button was depressed. This information was provided by the internal sampling code of the ASL system. In addition, peak instantaneous velocity and acceleration were identified. The sample frames for these peaks were then used to determine the time spent before and after peak velocity and acceleration, as well as the proportion of the total movement time spent before and after peak velocity and acceleration (cf. Chua \& Elliott).

Several points were highlighted in each of the velocity and acceleration profiles, and these helped to identify the kinematic indices of movement modifications. These were focused on the significant deviations in the acceleration trace in the period between peak velocity and the end of the movement. To identify these, a search was performed for any reversal points other than that of peak negative acceleration. If such a reversal point was found, the second reversal point following peak velocity was also marked. To be classed as a movement modification, two criteria had to be met. First, the amplitude between this marked reversal and the subsequent one had to be greater than the predefined amplitude criterion ( $14 \%$ of the greatest absolute magnitude of acceleration). Second, the duration between the marked reversal and the point at which the magnitude of acceleration fell below the value at the marked rever- 
sal had to equal or exceed a temporal criterion ( $72 \mathrm{msec}$; $\mathrm{cf}$. Chua \& Elliott, 1993; van Donkelaar \& Franks, 1991). This translates to five and nine sample frames at the $60-$ and $120-\mathrm{Hz}$ sampling frequencies, respectively.

Point of gaze tracking: ASL $\mathbf{- 4 1 0 0}$ analysis. The raw eye movement displacement data were analyzed according to previous work (Helsen, Starkes, \& Buekers, 1997). In the case of $60-\mathrm{Hz}$ data, a fixation started when 6 sequential gaze coordinate samples had a standard deviation less than or equal to $1^{\circ}$. In the case of $120-\mathrm{Hz}$ data, 12 sequential samples were required to have a standard deviation less than or equal to $1^{\circ}$. When these criteria were met, the fixation was considered to have started with the first of these samples, and the temporary fixation coordinates were the average vertical and horizontal coordinates for this initial group of samples. A fixation ended when 3 sequential gaze coordinate samples were all farther away from the temporary fixation than $1.25^{\circ}$. When this occurred, the last sample within $1.25^{\circ}$ was considered to be the end of the fixation. If sequences of 1 or 2 samples exceeded $1.25^{\circ}$ and were still considered within the same fixation, these samples were used to help calculate final fixation coordinates only if they did not exceed $1.5^{\circ}$. Because the movements of interest were purely horizontal, the algorithm focused only on the horizontal gaze coordinate samples.

Figure 2 illustrates what happens both temporally and spatially in a typical $120-\mathrm{Hz}$ trial. The time course of changes for both the PG and the hand ( $x$-axis) are shown. Along the $y$-axis, movement displacement from the home to the target position is shown. The plotted small circles are PG raw horizontal data samples. The superimposed flat lines represent each fixation. As can be seen from Figure 2, this algorithm provided a nice fit to the raw data and revealed first a 250-msec initiation time for PG, followed by a saccade which undershot the target (108-msec duration). A significant corrective saccade brought the target properly onto the fovea and resulted in a $200-\mathrm{msec}$ fixation. Using this algorithm, the occurrence of saccades larger than about $1.25^{\circ}$ of visual angle were reliably detected.

The integration of hand movements was made by an internal sampling code. This code marked the frames at which the light behind the home button went out (stimulus offset), the subject's finger left home position (initiation time hand-i.e., $308 \mathrm{msec}$ ), and finally, the subject pressed the target button (response time handi.e., $775 \mathrm{msec}$ ).

\section{Dependent Measures}

Performance measures. The primary index of overall performance was total response time of the hand. Initiation and movement times of both the hand and the PG also served as dependent measures. It should be noted that total response time was a composite of the other dependent measures: initiation time plus movement time equaled response time.

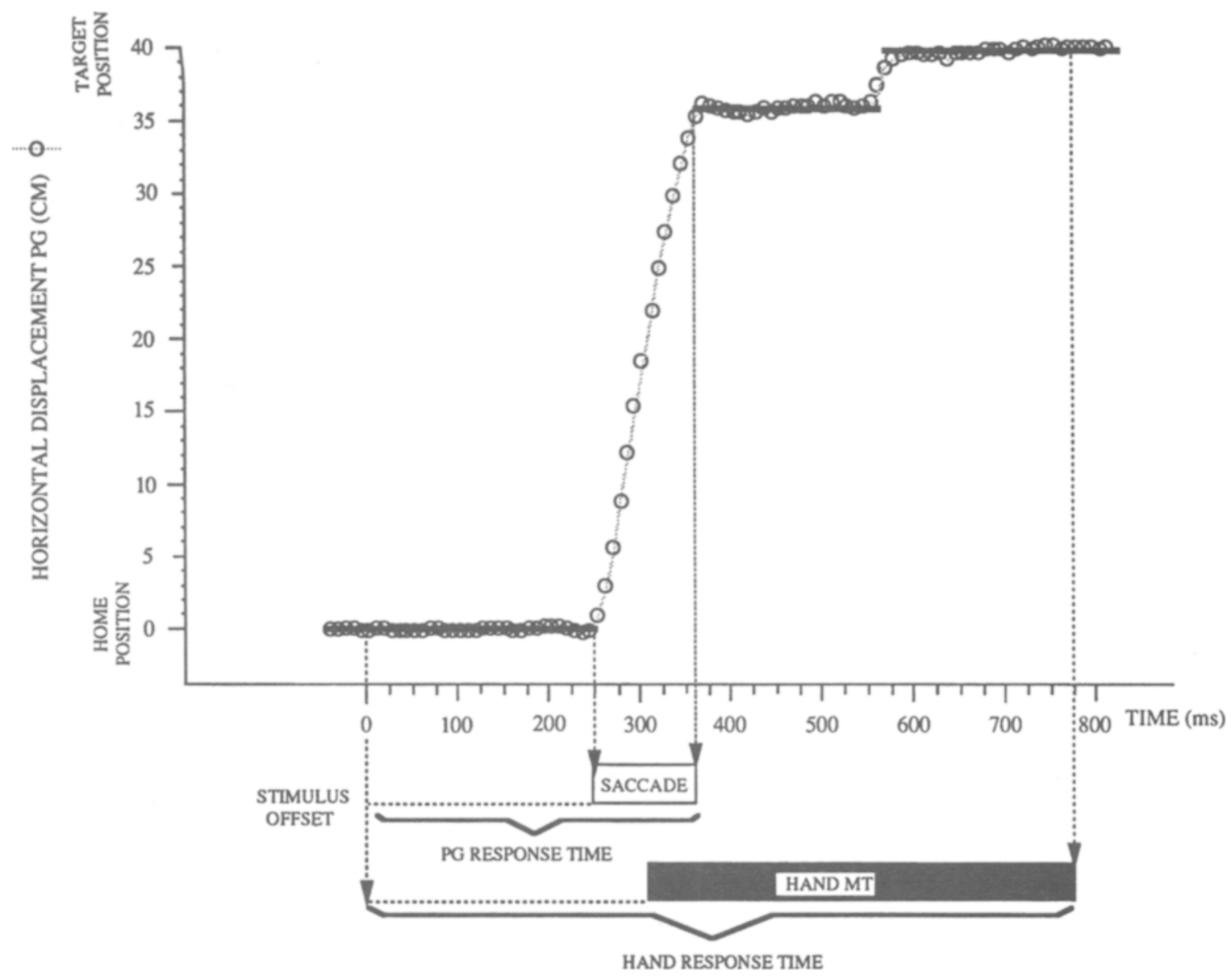

Figure 2. A representative spatiotemporal profile of point of gaze and hand movements for a typical trial in the 120-Hz condition. 
In addition to these measures of performance, some specific parameters were taken into account for finger and $P G$ movements separately, as well as for their interaction.

Finger kinematics. Kinematic data were available relating to peak velocity of the movement (PV), peak acceleration (PA), time to peak velocity (TTPV), time to peak acceleration (TTPA), time to peak velocity as a proportion of the movement time (PPV), and time to peak acceleration as a proportion of the movement time (PPA). In addition, data pertaining to movement modifications included the number of accelerations in the profile following peak velocity.

Point of gaze tracking. In addition to the basic initiation, movement, and response times for $\mathrm{PG}$, some specific parameters were also taken into consideration to compare the profile of aiming in this experiment with previous data (Helsen et al., 1997). To evaluate the variability within a subject across trials and experimental conditions, the frequency of occurrence of the various temporal onset and termination patterns of hand and PG movements was tallied. Saccade angle revealed the distance covered by each saccade. Fixation duration revealed how long subjects processed information after each saccadic movement. The number of saccades provided information on how consistently subjects made one or more saccades before pressing the target button.

The coordination of $P G$ and finger movements. One way of examining the significance of endpoint information for temporal organization has been to examine proportional time. Proportional time is the percentage of total response time of the hand elapsed when PG arrives on target. The present study focuses on two questions. First, how does proportional time relate to the kinematics of the finger and what is the effect of practice? Second, how will arrival time of the eye on target (response time PG derived from the ASL analyses) compare to TTPV and TTPA of the finger movement (derived from velocity and acceleration profiles generated from the Optotrak analyses)?

\section{Design}

All data were analyzed using separate analyses of variance (ANOVAs; randomized block factorial $2 \times 5$ design) for each of the dependent variables. Two sampling frequencies $(60 \mathrm{~Hz}, 120 \mathrm{~Hz})$ under five acquisition blocks (five blocks of five trials) were considered. The sources of any significant effects were identified through Newman-Keuls post hoc procedures $(p<.05)$. For significant interactions, simple main effects were analyzed using $F$ tests. For the number of accelerations, only a dependent measures $t$ test was completed comparing data from the 60 - and $120-\mathrm{Hz}$ sampling frequencies. All statistical tests were completed with alpha set at $p<.05$. Although there is the threat of inflation of alpha from numerous analyses conducted on data from the same subjects, in this case inflation of alpha provides a more stringent test. Any differences with sampling frequency should be more apparent; thus, nonsignificant effects are less likely. Guarding against Type II errors is of greater importance.

\section{RESULTS}

\section{Temporal Organization of PG and Finger Movements}

Table 1 presents summary information for most of the dependent measures acquired with both $60-$ and $120-\mathrm{Hz}$ sampling.

Initiation times. The analysis of initiation time data for both the hand and the PG showed no significant main effects. The acquisition block $\times$ sampling frequency interaction also was not significant.

To assess the integration of both the Optotrak fingertracking system and the ASL eye-tracking system, a correlation was calculated within each subject across trials to determine the relationship between initiation of the hand in the Optotrak and ASL systems. The mean correlations were as follows: $60 \mathrm{~Hz}(r=.98)$ and $120 \mathrm{~Hz}(r=.98)$. Since these correlations were all significant $(p<.01)$, initiation latencies of the hand were similar, regardless of sampling fre-

Table 1

Mean Values (in Milliseconds) and Standard Deviations of All Dependent Measures Sampled at $60 \mathrm{~Hz}$ and $120 \mathrm{~Hz}$

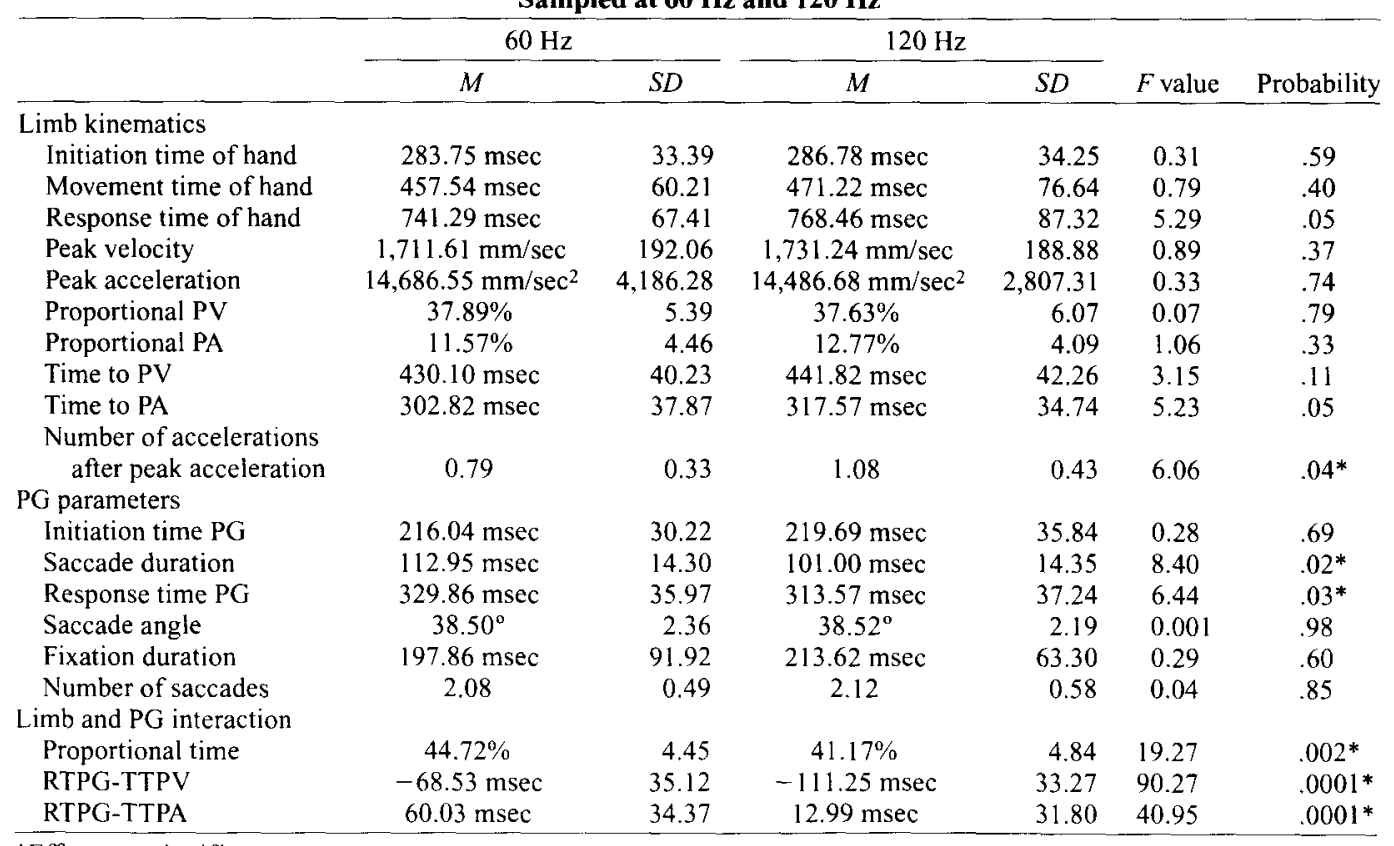

*Effect was significant. 
quency. The time difference averaged $36 \mathrm{msec}$, which probably resulted from an additional mechanical delay in the release of the home button $(60 \mathrm{~Hz}, M=38 \mathrm{msec} ; 120 \mathrm{~Hz}$, $M=34 \mathrm{msec}$ ). As would be expected, this constant was apparent in initiation times recorded by the ASL system but not in those recorded by the Optotrak system.

To evaluate the variability within a subject across trials and experimental conditions, the frequency of occurrence of the various temporal onset patterns of hand and PG movements was tallied. As can be seen from Table 2, for each of the two sampling frequencies, the subjects routinely initiated PG first, followed by the hand.

Movement times. The analysis of movement time data for the hand showed only a significant main effect of acquisition block $[F(4,36)=5.21, p<.002]$. NewmanKeuls post hoc tests showed that there was a significant decrease in hand movement time from Block $1(500 \mathrm{msec})$ to Blocks 2 (466 msec), 3 ( $454 \mathrm{msec}), 4$ (459 msec), and 5 (443 msec).

The analysis of movement time data for PG showed only a significant main effect of sampling condition $[F(1,9)=8.39, p<.0177]$, with saccade duration being longer at the lower sampling frequency $(60 \mathrm{~Hz}, M=$ $113 \mathrm{msec} ; 120 \mathrm{~Hz}, M=101 \mathrm{msec}$ ).

Response times. The analysis of response time data for the hand showed only a significant main effect of acquisition block $[F(4,36)=7.769, p<.0001]$. Post hoc tests showed a decrease in hand response time from Block 1 $(M=805 \mathrm{msec})$ to Blocks $2(M=746 \mathrm{msec}), 3(M=$ $742 \mathrm{msec}), 4(M=750 \mathrm{msec})$, and $5(M=732 \mathrm{msec})$. This essentially mirrors the movement time effect.

The analysis of response time data for PG showed only a significant main effect of sampling condition $[F(1,9)=$ $6.443, p<.0318]$. Specifically, there was a decrease in $\mathrm{PG}$ response time with increasing sampling frequency $(60 \mathrm{~Hz}, M=330 \mathrm{msec} ; 120 \mathrm{~Hz}, M=314 \mathrm{msec})$.

\section{Finger Kinematics}

The analyses of PV, PA, and PPA data for the finger showed no significant main effects. The acquisition block $X$ sampling frequency interactions also were not significant. In relation to the actual movement time of the finger, PPV data showed a significant main effect of acquisition block $[F(4,36)=2,64, p<.0495]$. Post hoc tests showed that there was a significant increase in PPV from Block $1(36 \%)$ to Block $5(40 \%)$. The differences between the other acquisition blocks were not significant $(2, M=$ $37 \% ; 3, M=38 \% ; 4, M=38 \%$ ).

Table 2

Frequencies (\%) for the Initiation Orders of Point of Gaze (PG) and Hand $(\mathrm{H})$ at Each Sampling Frequency (i.e., PG Prior to

Hand $[\mathrm{PG}<\mathrm{H} \mid$, Hand Prior to PG $[\mathrm{H}<\mathrm{PG}]$, and Hand Equal to $P G$ [ $\mathbf{H}=\mathbf{P G}]$ )

\begin{tabular}{rccc}
\hline Sampling Frequency & $\mathrm{PG}<\mathrm{H}$ & $\mathrm{H}<\mathrm{PG}$ & $\mathrm{H}=\mathbf{P G}$ \\
\hline $60 \mathrm{~Hz}$ & 98.40 & 0.80 & 0.80 \\
$120 \mathrm{~Hz}$ & 99.20 & 0.40 & 0.40 \\
\hline
\end{tabular}

The analysis of TTPV data showed only a significant main effect of acquisition block $[F(4,36)=2.79, p<.04]$. Post hoc tests showed that there was a significant decrease in TTPV from Block $1(M=454 \mathrm{msec})$ to Blocks $2(M=$ $430 \mathrm{msec}), 3(M=428 \mathrm{msec}), 4(M=433 \mathrm{msec})$, and 5 $(M=435 \mathrm{msec})$.

The analysis of TTPA data showed only a main effect of acquisition $[F(4,36)=3.24, p<.02]$. Post hoc tests showed that there was a significant decrease in TTPA from Block 1 (331 msec) to Blocks 2 (310 msec), 3 (301 msec), $4(302 \mathrm{msec})$, and $5(307 \mathrm{msec})$.

The analysis of the number of accelerations showed a main effect of sampling condition $[F(1,9)=6.06, p<$ $.04]$. Specifically, there was an increase in number of deviations with increased sampling frequency $(60 \mathrm{~Hz}, M=$ $20 ; 120 \mathrm{~Hz}, M=27)$.

\section{Point of Gaze Parameters}

The analysis of the visual angle covered by the first saccade showed only a main effect of acquisition block $[F(4,36)=3.11, p<.027]$. Post hoc tests revealed a significant decrease in visual angle from Block $2(M=$ $\left.38.93^{\circ}\right)$ to Block $5\left(M=37.97^{\circ}\right)$. The differences between Blocks $1\left(M=38.59^{\circ}\right), 3\left(M=38.61^{\circ}\right)$, and $4(M=$ $38.45^{\circ}$ ) were not significant. The analysis of fixation duration after the first saccade showed no significant main effects. The acquisition block $\times$ sampling frequency interaction also was not significant. On average, the subjects fixated for $207 \mathrm{msec}$. The analysis of the number of saccades showed no significant main effects. The acquisition block $\times$ sampling frequency interaction also was not significant. On average, the subjects made two saccades per trial.

\section{Coordination Parameters Between PG and the Hand}

Proportional time. The analysis of proportional time data showed a main effect of sampling condition $[F(1,9)=$ $19.27, p<.0017]$ and acquisition block $[F(4,36)=4.38$, $p<.0055]$ but no acquisition block $\times$ sampling frequency interaction. In terms of sampling frequency, there was a significant decrease in proportional time with increased sampling frequency $(60 \mathrm{~Hz}, M=45 \% ; 120 \mathrm{~Hz}, M=$ $41 \%$ ). Post hoc tests of the acquisition block effect revealed a significant increase in proportional time from Block 1 $(M=41 \%)$ to Blocks $3(M=43 \%), 4(M=43 \%)$, and 5 $(M=45 \%)$. As can be seen from Figure 3, because of the nonsignificant interaction effect, it was clear that, in both sampling frequencies, proportional time was moving toward approximately $50 \%$, as was originally seen in Helsen et al. (1997).

The analysis of time differences between response time PG and TTPA showed a main effect of sampling condition $[F(1,9)=40.95, p<.0001]$ and acquisition block $[F(4,36)=4.36, p<.0056]$ but no acquisition block $\times$ sampling frequency interaction. In terms of sampling frequency, there was a significant decrease in the time dif- 


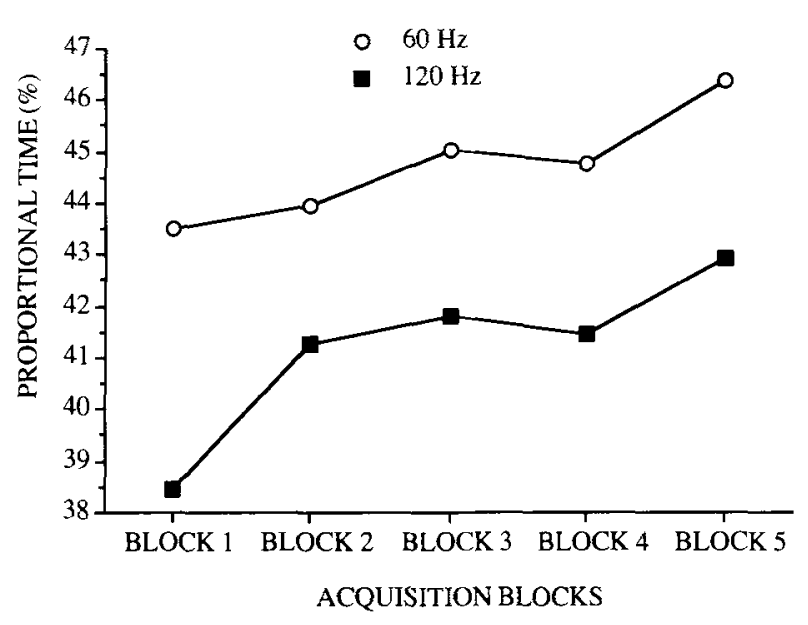

Figure 3. Proportional time (the ratio of point of gaze and hand response times) in each condition and acquisition block.

ference with increased frequency $(60 \mathrm{~Hz}, M=60 \mathrm{msec}$; $120 \mathrm{~Hz}, M=-13 \mathrm{msec}$ ). Post hoc tests of the acquisition block effect revealed a significant increase from Block 1 $(M=20 \mathrm{msec})$ to Blocks $2(M=32 \mathrm{msec}), 3(M=$ $44 \mathrm{msec}), 4(M=43 \mathrm{msec})$, and $5(M=43 \mathrm{msec})$.

The analysis of time differences between response time PG and TTPV showed a main effect of sampling condition $[F(1,9)=90.27, p<.0001]$ and acquisition block $[F(4,36)=3.26, p<.0223]$ but no acquisition block $\times$ sampling frequency interaction. In terms of sampling frequency, there was a significant increase in the time difference with increased frequency $(60 \mathrm{~Hz}, M=-68 \mathrm{msec}$; $120 \mathrm{~Hz}, M=-111 \mathrm{msec}$ ). Post hoc tests of the acquisition block effect revealed a significant decrease from Block 1 $(M=-103 \mathrm{msec})$ to Blocks $2(M=-87 \mathrm{msec}), 3(M=$ $-83 \mathrm{msec}), 4(M=-87 \mathrm{msec})$, and $5(M=-88 \mathrm{msec})$.

\section{A Profile of Typical PG and Hand Responses Across Sampling Frequencies}

Table 3 shows the mean values (in milliseconds) and standard deviations of primary and secondary eye movements for each sampling frequency. Saccadic eye movements were observed on all 500 trials, even though the subjects were not directly instructed to move their eyes.
In general, the primary saccadic eye movement started approximately $70 \mathrm{msec}$ prior to initiation of the hand movement. Saccade dynamics were in the range previously reported for duration/amplitude relationships (Bahill \& Stark, 1979; Jürgens, Becker, \& Kornhuber, 1981; Vercher, Magenes, Prablanc, \& Gauthier, 1994). These data demonstrate quite clearly that subjects generally produced saccades directly to the target region.

As can be seen from Table 3, in the majority of instances $(79 \%)$, the initial saccade was followed by a second saccade that brought the fovea exactly onto the target. This corrective saccade followed within a short latency, was typically small (less than $10 \%$ of primary saccade amplitude), and was of short duration. These properties of the oculomotor system have also been found elsewhere (Becker \& Fuchs, 1969). In both the 60- and the 120-Hz conditions, primary saccades usually undershot the target slightly ( $77 \%$ and $76 \%$ of the trials, respectively).

Given that the minimum time to process visual information is between 100 and $190 \mathrm{msec}$ (Carlton, 1981; Elliott \& Allard, 1985; Keele \& Posner, 1968; Zelaznik, Hawkins, \& Kisselburgh, 1983), information about target position was available well in advance of target acquisition, thus providing adequate time $(168 \mathrm{msec})$ for at least one visually based correction.

Recall that the results in both conditions were in line with the findings of previous experiments (Helsen et al., 1997), in terms of PG and hand initiation and response times. Changing sampling frequency altered only saccade duration and related parameters, not any of the other kinematic, PG, or coordination parameters.

\section{DISCUSSION}

When PG and hand movement data are sampled at $120 \mathrm{~Hz}$, information is recorded every $8.33 \mathrm{msec}$. At 60$\mathrm{Hz}$ sampling frequency, spatial coordinates are recorded every $16.66 \mathrm{msec}$. Intuitively, it would seem that, for optimal tracking of temporal and spatial information, a faster sampling frequency should be better (given the likelihood of very fast changes in acceleration for example). Presumably, a higher sampling frequency would permit more precise measurement of temporal features and, perhaps, evidence for a greater number of movement modifications,

Table 3

Mean Values and Standard Deviations of Primary and Secondary Eye Movements Recorded Using 60- and 120-Hz Sampling Frequencies

\begin{tabular}{|c|c|c|c|c|c|c|c|c|c|c|c|}
\hline \multirow[b]{2}{*}{ Sampling Frequency } & \multicolumn{2}{|c|}{ Initiation } & \multirow[b]{2}{*}{ Frequency } & \multicolumn{2}{|c|}{$\begin{array}{c}\text { Amplitude } \\
\text { (Visual Angle) }\end{array}$} & \multicolumn{2}{|c|}{$\begin{array}{c}\text { Saccade } \\
\text { Duration Time } \\
\end{array}$} & \multicolumn{2}{|c|}{$\begin{array}{l}\text { Response } \\
\text { Time PG }\end{array}$} & \multicolumn{2}{|c|}{$\begin{array}{c}\text { Proportional } \\
\text { Time } \\
\end{array}$} \\
\hline & $M$ & $S D$ & & $M$ & $S D$ & $M$ & $S D$ & $M$ & $S D$ & $M$ & $S D$ \\
\hline \multicolumn{12}{|l|}{$60 \mathrm{~Hz}$} \\
\hline Primary eye movement & $216 \mathrm{msec}$ & 30 & $100^{*}$ & $38.50^{\circ}$ & 2 & $113 \mathrm{msec}$ & 14 & $330 \mathrm{msec}$ & 36 & $45 \%$ & 4 \\
\hline Secondary eye movernent & & & $79 \dagger$ & $2.8^{\circ}$ & 0.8 & $19 \mathrm{msec}$ & 3 & $499 \mathrm{msec}$ & 52 & $67 \%$ & 6 \\
\hline \multicolumn{12}{|l|}{$120 \mathrm{~Hz}$} \\
\hline Primary eye movement & $220 \mathrm{msec}$ & 36 & $100^{*}$ & $38.52^{\circ}$ & 2 & $101 \mathrm{msec}$ & 14 & $314 \mathrm{msec}$ & 37 & $42 \%$ & 5 \\
\hline Secondary eye movement & & & $79 \dagger$ & $3.5^{\circ}$ & 0.9 & $10 \mathrm{msec}$ & 4 & $496 \mathrm{msec}$ & 60 & $64 \%$ & 7 \\
\hline
\end{tabular}

*Percentage of total trials $(N=250)$ containing at least one saccade. ${ }^{\ddagger}$ Percentage of total trials $(N=250)$ containing at least two saccades. 
and so forth. However, our results suggest that, even for a simple manual aiming movement done "as fast as possible," sampling data at $120 \mathrm{~Hz}$ showed very little advantage over that at $60 \mathrm{~Hz}$. For most of the dependent measures considered in this study -including initiation time, movement time, and response time of PG and the hand; time to peak velocity and acceleration of the hand; proportional time to peak velocity and acceleration of the hand; number of fixations of $\mathrm{PG}$-no significant differences occurred as a result of sampling frequency. Whether these results would generalize to more complex movements or other eye-hand tasks is unknown.

Where sampling frequency did have a significant effect was in the number of hand accelerations $(60 \mathrm{~Hz}$, $M=20 ; 120 \mathrm{~Hz}, M=27$ ), as well as in saccade duration of the primary saccade $(60 \mathrm{~Hz}, M=113 \mathrm{msec} ; 120 \mathrm{~Hz}$, $M=101 \mathrm{msec}$ ) and other derived parameters - such as response time of $\mathrm{PG}(60 \mathrm{~Hz}, M=330 \mathrm{msec} ; 120 \mathrm{~Hz}$, $M=314 \mathrm{msec})$ and proportional time $(60 \mathrm{~Hz}, M=$ $45 \% ; 120 \mathrm{~Hz}, M=41 \%$ ) - in which saccade duration was one underlying component. With saccade duration, a $60-\mathrm{Hz}$ sampling frequency appears to overestimate saccade duration basically by 1.5 samples $(12 \mathrm{msec})$. The higher sampling frequency probably measures both initiation and termination of PG more precisely. At this point, it is unknown whether saccade duration might be further reduced with even higher sampling frequencies. Since acceleration changes may occur quite rapidly, the higher sampling frequency may render more of these changes measurable. Very often number of accelerations is used to represent the number of visually based corrections made during the course of a movement (Chua \& Elliott, 1993). For this reason, when modeling the open versus closed loop nature of movement, it would be preferable to derive acceleration profiles on the basis of at least a $120-\mathrm{Hz}$ sampling frequency.

Recall that one issue in sampling both eye and hand movements is the synchronization of data collection between two systems, given that the Optotrak records limb movement and the ASL 4100 records PG movements. In this study, a Pearson product-moment correlation was calculated between hand initiation times measured by the two systems to determine any discrepancies. The correlation was .98 , and the unexplained variability might be due to the ASL system's being triggered by the release of the home button. The small time difference of $36 \mathrm{msec}$ between the two systems most likely was the result of the additional mechanical time delay of the button release.

Finally, beyond affecting precision of measurement of many of the dependent measures, sampling frequency could potentially have influenced the acquisition findings. For example, a higher sampling frequency might have picked up significant learning effects after fewer trials than did a lower sampling frequency. This proved not to be the case. There were no cases in which the effects of acquisition block interacted with sampling frequency.
The results of this study would seem to be good news for those researchers who currently have $60-\mathrm{Hz}$ ASL systems. Depending on which dependent measures are being assessed, data from the lower sampling frequency systems may be quite equivalent to that afforded by the higher frequency, and also higher priced, system. On the down side, if saccade duration of the primary or subsequent saccades are of particular import, the researcher would be well advised to consider the advantages of higher frequency sampling. In terms of limb kinematics, although $60 \mathrm{~Hz}$ seems sufficient for describing the primary acceleration and deceleration of the hand, a higher sampling frequency appears to be necessary to identify subsequent corrective submovements that are of a shorter duration and a smaller amplitude.

\section{REFERENCES}

BAHILl, A. T., \& STARK, L. (1979). The trajectories of saccadic eye movements. Scientific American, 240, 108-117.

BeCKER, W., \& FuCHS, A. F. (1969). Further properties of the human saccadic system. Vision Research, 9, 1247-1259.

Carlton, L. G. (1981). Processing visual feedback information for movement control. Journal of Experimental Psychology: Human Perception \& Performance, 7, 1019-1030.

ChuA, R., \& ElliotT, D. (1993). Visual regulation of manual aiming. Human Movement Science, 12, 365-401.

ELLIOTT, D., \& AllaRD, F. (1985). The utilization of visual feedback information during rapid pointing movements. Quarterly Journal of Experimental Psychology, 37A, 407-425.

FITTS, P. M. (1954). The information capacity of the human motor system in controlling the amplitude of movement. Journal of Experimental Psychology, 47, 381-391

Helsen, W. F., Starkes, J. L., \& Buekers, M. J. (1997). Effects of target eccentricity on temporal costs of point of gaze and the hand in aiming. Motor Control, 1, 161-177.

Jürgens, R., Becker, W., \& KornHuber, H. H. (1981). Natural and drug-induced variations of velocity and duration of human saccadic eye movements: Evidence for a control of the neural pulse generator by local feedback. Biological Cybernetics, 39, 87-96.

Keele, S. W., \& Posner, M. I. (1968). Processing of visual feedback in rapid movements. Journal of Experimental Psychology, 77, 155-158.

RADAU, P., TwEED, D., \& VILIS, T. (1994). Three-dimensional eye, head, and chest orientations after large gaze shifts and the underlying neural strategies. Journal of Neurophysiology, 72, 2840-2852.

RoBinson, D. A. (1963). A method of measuring eye movement using a scleral search coil in a magnetic field. IEEE Transactions on Biomedical Engineering, 10, 137-145.

van Donkelaar, P., \& Franks, I. M. (1991). The effects of changing movement velocity and complexity on response preparation: Evidence from latency, kinematic, and EMG measures. Experimental Brain Research, 83, 618-632.

Vercher, J. L., Magenes, G., Prablanc, C., \& Gauthier, G. M. (1994). Eye-head-hand coordination in pointing at visual targets: Spatial and temporal analysis. Experimental Brain Research, 99, 507-523.

YOUNG, L. R., \& SHEENA, D. (1975). Survey of eye movement recording methods. Behavior Research Methods \& Instrumentation, 7, 397-429.

ZelaZNiK, H. N., HaWkINS, B., \& KisselbuRGH, L. (1983). Rapid visual feedback processing in single-aiming movements. Journal of Motor Behavior, 15, 217-236.

(Manuscript received December 10, 1996; revision accepted for publication April 18, 1997.) 